\title{
Fabrication of carbon black- and graphene-supported PtSn electrocatalyst for glucose oxidation: electrochemical and surface studies
}

\author{
Mark Kenneth C. Gonzales ${ }^{1,2}$, Hao Yuhn C. Hsu ${ }^{1,3}$, Harris M. Fulo ${ }^{1,3}$, \\ E Bernard John V. Tongol ${ }^{1,2,3 *}$ \\ ${ }^{1}$ Research Center for the Natural and Applied Sciences \\ ${ }^{2}$ Department of Chemistry, College of Science; ${ }^{3}$ The Graduate School \\ University of Santo Tomas, España Boulevard, 1015 Manila, PHILIPPINES
}

\begin{abstract}
The study aimed to prepare and characterize carbon black- and graphene-supported PtSn catalysts as possible electrocatalysts alternative for glucose oxidation. Carbon-based PtSn catalysts were successfully synthesized by the borohydride-facilitated reduction method as confirmed by energy dispersive X-ray spectroscopy (EDX) and X-ray diffraction (XRD) analyses. The XRD pattern also revealed the formation of PtSn nanoparticles of less than $5 \mathrm{~nm}$ size. The electro-oxidation of glucose on PtSn/C was studied by cyclic voltammetry at a potential range from $-1.0 \mathrm{~V}$ to $0.6 \mathrm{~V}$ (vs. Ag/AgCl). Three oxidation peaks at $-0.8 \mathrm{~V},-0.3 \mathrm{~V}$, and $0.05 \mathrm{~V}$, which correspond to the oxidation processes of glucose, were observed. The addition of a secondary metal, Sn, showed an enhanced catalytic activity of Pt towards glucose electro-oxidation. The optimization of metal loading and metal ratio using carbon black (XC-72) as support was studied and the results revealed that the optimum metal loading is $40 \%$ while the optimum PtSn ratio is 1:0.01 using the $40 \%$ metal loading. The lower amount of Sn added can lower the poisoning effect of Pt and thus only act as a promoter. The optimized parameters for carbon black were used for graphene as support. The data revealed that graphene as a support has an enhanced electrocatalytic activity compared to carbon black with a peak current of $12.82 \mathrm{~mA} / \mathrm{cm}^{2}$ and $6.97 \mathrm{~mA} / \mathrm{cm}^{2}$, respectively. The XRD data indicates that graphene is a promising support material that could modify the catalytic properties of PtSn bimetallic electrocatalyst for fuel cell catalysis.
\end{abstract}

Keywords: Pt/Sn nanoparticles, PtSn/C, PtSn/graphene, glucose electro-oxidation

\section{INTRODUCTION}

With an increasing demand of pollution-free and cheap energy, researchers are focusing on more easily available, less toxic and safer fuel. This

*To whom correspondence should be addressed bvtongol@mnl.ust.edu.ph; bjvtongol@yahoo.com brings the researchers to the very advantageous concept of a fuel cell that could run on glucose. Glucose is a renewable and abundant energy source as it can be derived from a large amount of waste biomass that is generated by agricultural activities and from dedicated energy crops, such as corn and other grains [1]. 
A good approach to derive energy from glucose is to feed it to a fuel cell that directly oxidizes the fuel to generate electricity. However, glucose is a very stable compound and is difficult to oxidize electrochemically. Thus, catalysts have been developed to facilitate the electrooxidation of glucose. Noble metals, such as platinum, can potentially catalyze glucose oxidation, but their use has many drawbacks. Bimetallic catalysts were being explored for application in direct glucose fuel cells (DGFC). Different bimetallic electrocatalysts such as $\mathrm{PtBi}$, PtRu, PtPd, PtAu, etc. have showed catalytic activity towards glucose oxidation and produced higher current and power densities [1-4].

Bimetallic Au-Ag particles-modified on reduced graphene oxide electrodes catalyzed the electrochemical oxidation of glucose, showing a synergetic catalytic effect of Au and Ag [5]. However, the use of both metals is known to be costly.

Recent studies on anodes for glucose electrooxidation using PdSn in alkaline media introduced the effect of Sn as a secondary metal towards glucose oxidation [6]. Metal ratio was shown to be an important factor, $\mathrm{Pd}_{3} \mathrm{Sn}_{2} / \mathrm{C}$ metal ratio showing higher electrolytic activity compared to $\mathrm{PdSn} / \mathrm{C}$ and $\mathrm{Pd} / \mathrm{C}$. High amount of electrolyte and glucose, as well as a temperature at $40^{\circ} \mathrm{C}$, enhanced the rate of glucose electrooxidation.

The development of a catalyst support is highly advantageous for a good dispersion and utilization of the catalyst. The most common support matrix is carbon black (XC-72) since it has a higher surface area, cheaper, and can improve the catalytic activity of an electrocatalyst [7]. However, graphene, an allotrope of carbon, has exhibited unique and excellent properties as a catalyst support. Its use as support can provide the main requirements of suitable supports for fuel cell catalysts: high surface area, good electrical conductivity, suitable porosity to allow good reactant flux, and high stability in fuel cell environment [8]. The development of graphene as a support is encouraged by many researchers because it is less expensive than carbon nanotubes.

Huang and co-workers [9] demonstrated a facile and controllable approach to synthesize graphene nanoplate-Pt (GNP/Pt) composite and facilitate their application in fuel cell technologies. It has been found that GNP/Pt has better electrocatalytic activity and stability for methanol oxidation. The development of Pt-CuO nanoparticles decorated reduced graphene oxide gained an immense study on direct electrochemical activity for its application for a highly sensitive non-enzymatic disposable glucose sensor [10].

This paper describes the fabrication of graphenesupported PtSn composite as electrocatalyst towards glucose electro-oxidation. PtSn/ graphene and $\mathrm{PtSn} / \mathrm{C}$ composites are synthesized via borohydride-facilitated reduction, and their electrochemical and surface properties are compared using cyclic voltammetry and energy dispersive X-ray (EDX) and X-ray diffraction (XRD), respectively. As of this writing, there has been no study on PtSn bimetallic catalyst dispersed on graphene and carbon black for the electro-oxidation of glucose.

\section{EXPERIMENTAL}

Materials. Graphene synthesis includes graphite powder (Showa, Japan), $\mathrm{H}_{2} \mathrm{SO}_{4}$ (Scharlau, Spain), $\mathrm{NaNO}_{3}$ and $\mathrm{KMnO}_{4}$ (Merck, Germany). Carbon black Vulcan XC-72 (Cabot) was used as a support in the optimization process. $\mathrm{H}_{2} \mathrm{PtCl}_{6}$ (Sigma Aldrich, Singapore) and $\mathrm{SnSO}_{4}$ (Sigma Aldrich, Singapore) were used as platinum and tin precursors, respectively. $\mathrm{NaBH}_{4}$ $(98 \%+$ powder, Acros Organics) was used as a reducing agent. 
Aqueous solutions were prepared during catalyst preparation using ultrapure water (TOC $<5.00$ ppb; Resistivity = 18.2 M $2 \mathrm{~cm}^{-}$; Millipore Water Systems, USA). Nafion dispersion (Sigma Aldrich, Singapore) and iso-propanol (Merck, USA) were used for catalyst ink preparation. Solutions of D-glucose (Merck, USA) and $\mathrm{NaOH}$ (Merck, USA) were used as fuel and electrolyte, respectively.

\section{Fabrication of carbon black and graphene-} supported Pt/Sn composite. Graphene synthesis was done with natural graphite powder by a modified-Hummers and Offeman's method [11].

To co-deposit Pt and Sn on graphene, a certain volume (depending on the ratio of $\mathrm{Pt}$ and $\mathrm{Sn}$ ) of salt solutions of $0.02 \mathrm{M} \mathrm{H}_{2} \mathrm{PtCl}_{6}$ and $0.02 \mathrm{M}$ $\mathrm{SnSO}_{4}$ was added into the graphite oxide solution while stirring. Then, chemical reduction was performed by the addition of metal: $\mathrm{NaBH}_{4}$ with a 1:4 ratio onto the mixture to produce the $\mathrm{Pt} / \mathrm{Sn}$ metal and graphene composite. The resulting solid was washed with excess ethanol and ultrapure water to obtain the PtSn/graphene composite. The solid was vacuum-dried at $40^{\circ} \mathrm{C}$.

\section{Electrochemical characterization of Pt/Sn-} modified graphene. A solution consisting of $1.0 \mathrm{mg}$ of PtSn/graphene composite, $0.98 \mathrm{~mL}$ isopropanol and $0.02 \mathrm{~mL}$ of Nafion solution was prepared and sonicated for $15 \mathrm{~min}$. A total volume of $80 \mathrm{~mL}$ of the composite solution was drop-cast over the glassy carbon electrode (GCE) surface. The resulting electrode was dried in the oven at $60^{\circ} \mathrm{C}$ for $5 \mathrm{~min}$. Once dried, the electrode was connected to the CV set-up and placed into the CV cell. The electrocatalytic activity of the PtSn/graphene electrocatalyst towards glucose oxidation was investigated using cyclic voltammetry from $-1.0 \mathrm{~V}$ to $0.6 \mathrm{~V}$ at a scan rate of $20 \mathrm{mV} / \mathrm{s}$ for 10 cycles.

All electrochemical measurements were performed in a conventional 3-electrode electrochemical cell at room temperature. The solutions were purged by bubbling with high purity $\mathrm{N}_{2}$ gas for 15 min and under $\mathrm{N}_{2}$ atmosphere during electrochemical measurements. Pt rod and $\mathrm{Ag} / \mathrm{AgCl}$ (sat. $3 \mathrm{M} \mathrm{KCl}$ ) were used as the counter and reference electrode, respectively. The working electrodes were the fabricated carbon black- and graphene-supported PtSn composite. The electrochemical measurements were carried out using EDAQ potentiostat (Australia) under control of dedicated software (EChem, EDAQ). All potentials reported in this paper are presented in the $\mathrm{Ag} / \mathrm{AgCl}$ scale.

\section{Surface characterization of Pt/Sn-modified} graphene. Surface morphology and elemental analyses of $\mathrm{PtSn} / \mathrm{C}$ and $\mathrm{PtSn} / \mathrm{graphene}$ composites were recorded using a JEOL JSM 5310 scanning electron microscope (SEM) with an accelerating voltage of $15 \mathrm{keV}$ and energy dispersive X-ray (EDX) analysis, respectively. The X-ray diffraction analysis was done with Siemens Kristalloflex-76 X-ray generator using $\mathrm{Cu} \mathrm{Ka}(\lambda=0.15405 \mathrm{~nm})$ source and Philips 1080 vertical Goniometer. The source was operated with a tube current of $100 \mathrm{~mA}$ and tube voltage of $32 \mathrm{kV}$. The $2 \theta$ Bragg's angles were scanned over the range of $10-90^{\circ}$ and were explored at a scan rate of $5 \mathrm{~min}^{-1}$.

\section{Results AND DISCUSSION}

Glucose electro-oxidation on $\mathbf{P t} / \mathrm{C}$. Glucose electro-oxidation on $\mathrm{Pt} / \mathrm{C}$ substrate was studied using cyclic voltammetry with a potential range from $-1.0 \mathrm{~V}$ to $0.6 \mathrm{~V}$ in $0.5 \mathrm{M} \mathrm{NaOH}$ electrolyte. Figure 1 shows the three electro-oxidation peaks for $\mathrm{Pt} / \mathrm{C}$ electrocatalyst. The presence of three visible oxidation peaks from negative to slightly positive potential with respect to $\mathrm{Ag} / \mathrm{AgCl}$ (sat. $\mathrm{KCl}$ ) reference electrode indicates the occurrence of more than one electro-oxidation reactions. The three oxidation peaks can provide the information that glucose was oxidized electrochemically. 
The three very clear oxidation peaks observed for glucose electro-oxidation on $\mathrm{Pt} / \mathrm{C}$ is in agreement with those found in literature [1, 2, 6 , 9]. The anodic peak at $-0.8 \mathrm{~V}$ (labeled A in Fig. 1) shows the characteristic of the surface process of an adsorbed species. It appears due to chemisorption and dehydrogenation of glucose at very low potential. This peak coincides with the hydrogen desorption peak on platinum electrode. The dehydrogenated intermediate formed by electro-oxidation at $-0.8 \mathrm{~V}$ leads to formation of gluconate structure by successive steps. The anodic peak at $-0.3 \mathrm{~V}$ (labeled B in Fig. 1) appears at $\mathrm{OH}$ adsorbed catalyst surface by direct glucose oxidation from the bulk to lactone, which on hydrolysis produces gluconate. This is where the complete oxidation of glucose happens. The anodic peak at $0.05 \mathrm{~V}$ (labeled C in Fig. 1) is obtained on already oxidized catalyst surface and may be due to oxidation of adsorbed residues [1, 2, 9].

Glucose electro-oxidation on PtSn/C. The addition of Sn on the substrate produced an improved catalytic activity towards glucose electro-oxidation. The three oxidation peaks on $\mathrm{PtSn} / \mathrm{C}$ electrocatalyst showed a greater current density as shown in Fig. 1, indicative of the promoting effect of Sn on the catalytic activity of Pt.

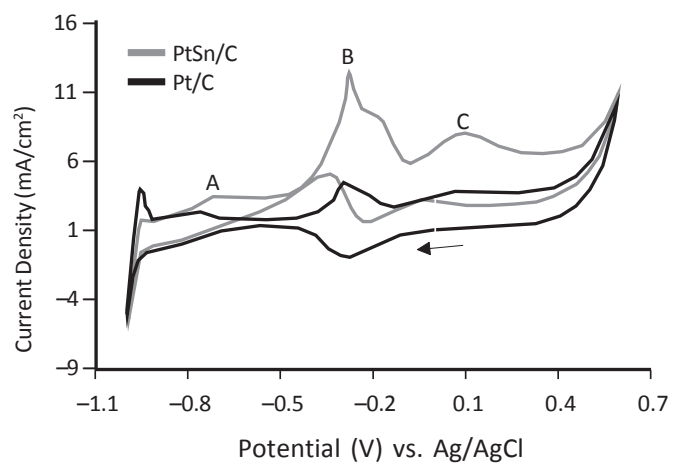

Figure 1. Glucose electro-oxidation on $\mathrm{Pt} / \mathrm{C}$ and $\mathrm{PtSn} / \mathrm{C}$, scan rate: $20 \mathrm{mV} / \mathrm{s}$
The addition of Sn into Pt can significantly improve the reaction rate but the amount of $\mathrm{Sn}$ to be added should be noted. The effects of Sn on Pt catalysts have been believed to be associated with modification of geometric and electronic structures by PtSn interactions, thus consequently leading to changes in catalytic activities [12]. The impurities that poison the surface Pt sites are partially decreased since Pt sites tend to be reduced as the surface Sn sites are readily oxidized to form tin oxide species.

The Sn addition modifies the electronic environment of PtSn catalysts by influencing the electron affinities of the surface Pt atoms and produces surface oxygenated species which can act as an oxidant source, thereby increasing stability through efficient oxidation of surface poisoning species.

Figure 2 presents the comparison of the integrated values of the three anodic peaks $\left(1^{\text {st }}\right.$ peak-white, $2^{\text {nd }}$ peak-black, $3^{\text {rd }}$ peak-gray) for glucose oxidation on $\mathrm{Pt} / \mathrm{C}$ and $\mathrm{PtSn} / \mathrm{C}$. The PtSn/ $\mathrm{C}$ showed a higher integrated current density, indicating that the addition of Sn caused an enhancement in the catalytic activity of $\mathrm{Pt}$ towards glucose oxidation.

Optimization of parameters. The changing of the metal loading affects the activity of the catalyst (Fig. 3). Increasing the metal loading

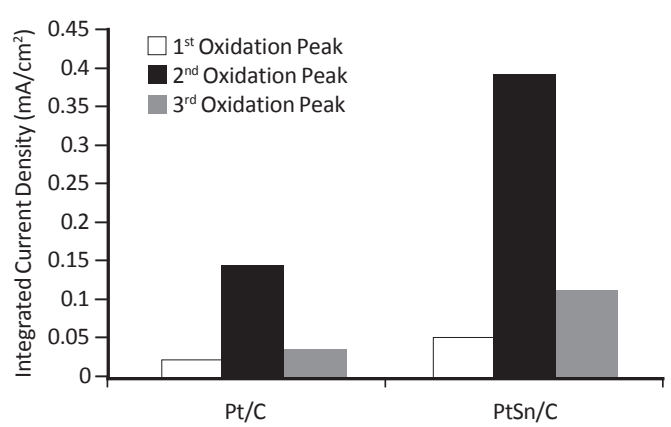

Figure 2. Integrated current density values of the three anodic peaks of glucose oxidation on $\mathrm{Pt} / \mathrm{C}$ and PtSn/C 


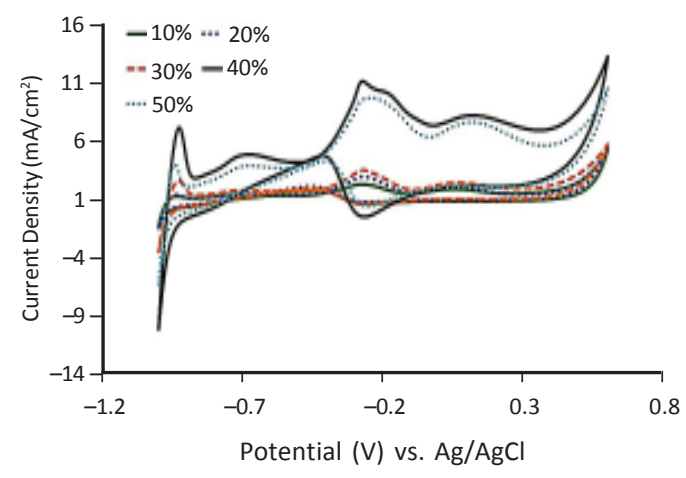

Figure 3.Cyclic voltammogram $\mathrm{PtSn} / \mathrm{C}$ at different metal loading using $10 \mathrm{mmol}$ glucose in $0.5 \mathrm{M}$ $\mathrm{NaOH}$, scan rate: $20 \mathrm{mV} / \mathrm{s}$

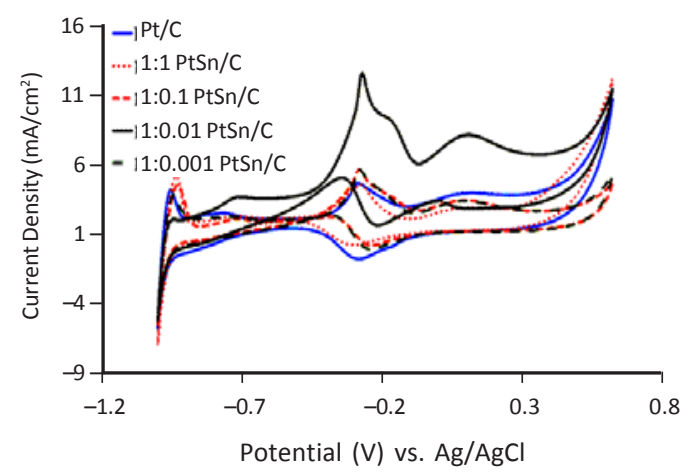

Figure 4. Cyclic voltammogram $\mathrm{PtSn} / \mathrm{C}$ at different metal ratio using $10 \mathrm{mmol}$ glucose in $0.5 \mathrm{M}$ $\mathrm{NaOH}$, scan rate: $20 \mathrm{mV} / \mathrm{s}$

increased the electrocatalytic activity of PtSn with 1:1 metal ratio. Metal loading of $10 \%, 20 \%$, and $30 \%$ demonstrated small oxidation peaks for glucose oxidation, compared to $40 \%$ and $50 \%$ metal loading. The $40 \%$ and $50 \%$ metal loading showed a large increase in the current density among the three anodic peaks in glucose electrooxidation.

The PtSn/C metal ratio influenced glucose oxidation, as shown in Fig. 4. When a smaller amount of Sn was added, a greater catalytic activity was obtained. A greater amount of Sn can lower the catalytic activity of Pt. Higher Sn content may seriously inhibit the dissociative

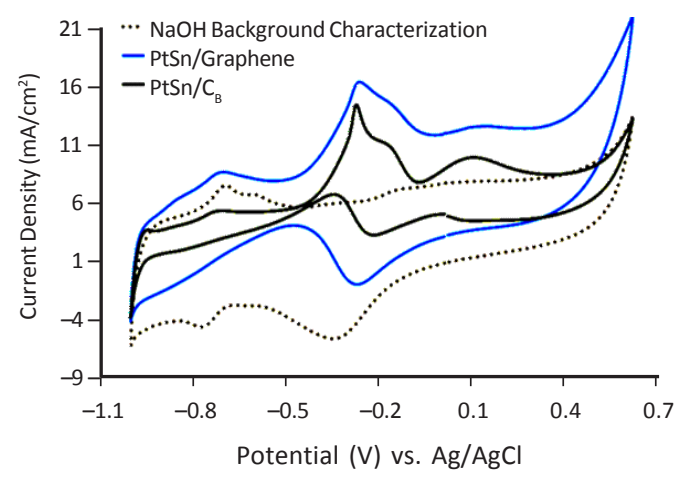

Figure 5.Glucose electro-oxidation on PtSn/C and $\mathrm{PtSn} /$ graphene, scan rate: $20 \mathrm{mV} / \mathrm{s}$

chemisorption of glucose due to too much diminished Pt ensembles size and a substantial reduction of available surface Pt active sites by larger amounts of Sn species [12].

The optimum PtSn metal ratio is 1:0.01. This composition can provide the suitable modification of Pt surface by Sn, which might be satisfied in terms of the changes in the electronic and geometric structures of Pt active sites for the corresponding glucose oxidation. At this ratio, the oxidation of glucose showed an increase of current density indicative of an improved catalytic activity of the PtSn catalyst towards glucose oxidation.

\section{Glucose electro-oxidation on graphene-} supported Pt/Sn composite. Glucose electro-oxidation on $\mathrm{PtSn}$ /graphene substrate was studied using cyclic voltammetry technique ranging from $-1.0 \mathrm{~V}$ to $0.6 \mathrm{~V}$ in $0.5 \mathrm{M} \mathrm{NaOH}$ electrolyte. The optimized parameters, which are 40\% metal loading and 1:0.01 Pt/Sn metal ratio for carbon black, were used for graphene as support. The data revealed that graphene as a support has an enhanced electrocatalytic activity compared to carbon black with a peak current of $12.82 \mathrm{~mA} / \mathrm{cm}^{2}$ and $6.97 \mathrm{~mA} / \mathrm{cm}^{2}$, respectively as shown in Fig. 5. 

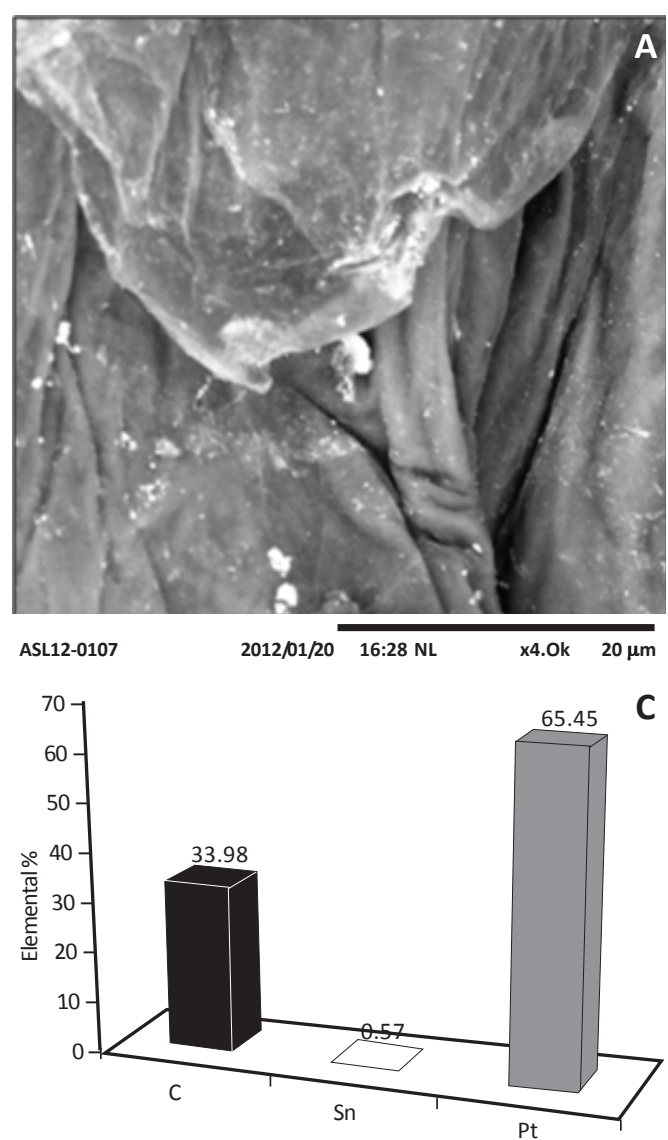

Figure 6. SEM images of (A) PtSn/graphene and (B) PtSn/C; EDX profile of (C) PtSn/graphene and (D) PtSn/C
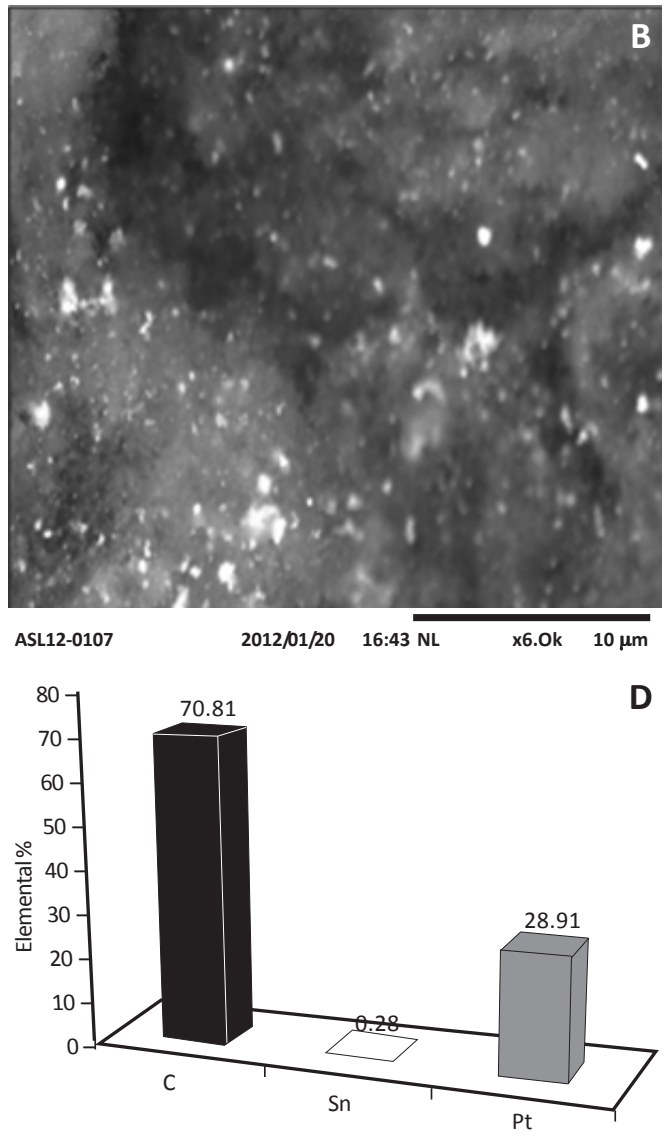

The effect of graphene support not only maximized the availability of $\mathrm{Pt} / \mathrm{Sn}$ electrocatalyst surface area for electron transfer but also provided better mass transport of reactants to the electrocatalyst [12].

Surface characterization. The SEM micrograph (Fig. 6a) reveals that graphene was successfully fabricated, displaying micrometer-long wrinkles, flake-like shapes and semi-translucent crumpled morphology characteristic of graphene sheet [14]. The Pt and Sn nanoparticles were poorly dispersed and distributed on the carbon black support (Fig. 6b). There was no indication of uniformity in the size and shape of the catalysts.
The EDX profile (Fig. 6c and d) confirmed the presence of elemental C, Pt and Sn with a 1:0.01 $\mathrm{Pt} / \mathrm{Sn}$ metal ratio. The presence of carbon and metal elements such as Pt and Sn and absence of other elements in EDX images indicate the successful reduction of metal precursors on carbon.

The EDX results indicated a greater Pt content for PtSn/graphene compared to PtSn/C. The enhancement of the catalytic activity of the PtSn/ graphene bimetallic electrocatalyst may be due to the greater Pt content.

Figure 7 shows the XRD patterns of the carbon black and graphene-supported with $40 \%$ wt and 1:0.01 PtSn composites. The characteristic peaks 


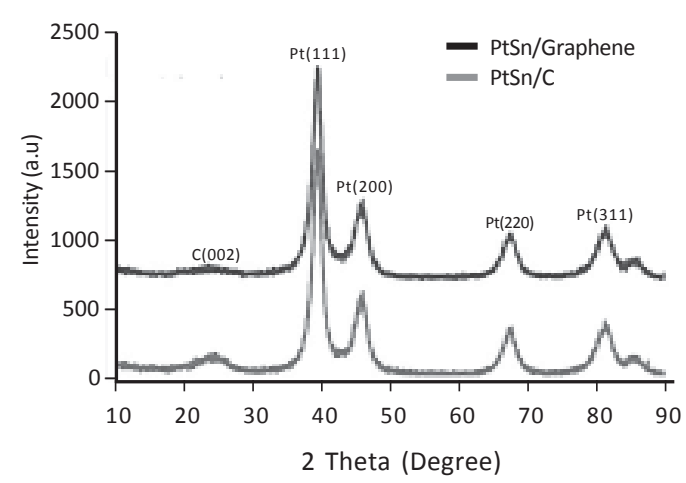

Figure 7. XRD profile of PtSn/C (gray) and PtSn/ graphene (black)

of a crystalline face centered cubic (fcc) Pt phase from (1 11 1), (2 0 0 0), (2 2 0), and (3 11 1) planes appeared at the corresponding diffraction angles. The first broad peak around $25^{\circ}$ is associated with $\left(\begin{array}{lll}0 & 0 & 2\end{array}\right)$ plane of the hexagonal structure of carbon support [12].

The typical fcc-Pt diffraction peaks in all PtSn/C catalysts appear to be broadened due to small particle size effect. There were no noticeable peaks for a pure $\mathrm{Sn}$ or its oxides in the XRD measurements, indicating a good degree of alloying between Pt and Sn although tin oxides may exist in its amorphous form. The amount of Sn added is also too small to have a distinct diffraction peak. The XRD data also revealed a more intense diffraction peak for carbon black compared to graphene. This is due to single sheets of graphene fabricated as revealed by SEM analysis.

The average particle sizes and lattice parameters for the single-phase Pt was evaluated from the full width at half maximum (FWHM) and the angular position (2 $\theta$ max) of the Pt (2 20 ) peaks using the Scherrer formula

$$
\mathrm{L}=0.94 \lambda_{\mathrm{Ka} 1} / \mathrm{B}_{2 \theta} \cos \theta_{\text {max }}
$$

where $\mathrm{L}$ denotes the average particle size, the value 0.94 comes from spherical crystallite geometry (cubo-octahedral shape), $\lambda_{\mathrm{Ka} 1}$ is the wavelength of $X$-ray radiation $(1.54056 \mathrm{~nm}), \theta_{\text {max }}$ is the angular position at the (2 20 ) peak maximum, and $\mathrm{B}_{(2 \theta)}$ is the full width at half maximum (FWHM) of the peak broadening in radians. The Pt (2 20 ) diffraction peak was used as the reference so as to avoid possible disturbances from the carbon support [13]. Based on the Scherrer formula, the average particle size of Pt in carbon black and graphenesupported Pt/Sn are 4.75 $\pm 0.05 \mathrm{~nm}$ and 4.07士 $0.05 \mathrm{~nm}$, respectively.

The Pt particles on graphene-supported composite are slightly smaller compared to $\mathrm{Pt}$ particles on carbon black-supported composite due to a good dispersion of Pt particles on the graphene layer. The smaller particle size contributes to a higher surface area and a consequent higher catalytic activity effect towards glucose oxidation. This behavior indicates that the unique structure and physical properties of graphene can be helpful in the distribution of Pt and Sn nanoparticles.

The interaction of Pt and Sn nanoparticles and graphene surface can provide a higher surface area for the Pt and Sn particles and exposing larger area of the Pt/Sn particles. As a result, charge transport and better mass transfer of the reactants are maximized. Graphene as a twodimensional nanomaterial has a high theoretical specific surface area which could be favorable for electrochemical reaction. On the other hand, Pt and Sn nanoparticles could be easily deposited on the surface of graphene by providing anchoring centers based on 2D flat planes and oxygen-containing groups [14].

\section{Conclusion}

The addition of Sn to Pt promotes the electrocatalytic activity towards glucose oxidation. A very small amount of Sn could improve the catalytic activity of Pt towards glucose oxidation by providing a higher current density. 
Graphene as support produced an enhanced electrocatalytic activity compared to carbon black. Smaller particles were formed when graphene was employed as the catalyst support. Graphene is indeed a promising support material that could modify the catalytic properties of PtSn bimetallic electrocatalyst for fuel cell catalysis.

\section{ACKNOWLEDGMENT}

This research work was financially supported by the UST-Research Center for the Natural and Applied Sciences (UST-RCNAS) and the Department of Science and Technology (DOST).

Scanning electron microscopy (SEM) and energy dispersive X-ray (EDX) analysis were conducted at the De La Salle University, Manila) analysis, respectively. The X-ray diffraction analysis was done at the Applied Physics Laboratory of the DOST-Philippine Nuclear Research Institute.

\section{REFERENCES}

[1] Basu D, Basu S. A study on direct glucose and fructose alkaline fuel cell. Electrochimica Acta 2010; 55(20):5775-5779.

[2] Basu D, Basu S. Synthesis, characterization and application of platinum based bi-metallic catalysts for direct glucose alkaline fuel cell. Electrochimica Acta 2011; 56(17):6106-6113.

[3] Basu D, Sood S, Basu S. Performance comparison of $\mathrm{Pt}-\mathrm{Au} / \mathrm{C}$ and $\mathrm{Pt}-\mathrm{Bi} / \mathrm{C}$ anode catalysts in batch and continuous direct glucose alkaline fuel. Chemical Engineering Journal 2013; 228:867-870.

[4] Basu D, Basu S. Synthesis and characterization of Pt-Au/C catalyst for glucose electro-oxidation for the application in direct glucose fuel cell. International Journal Hydrogen Energy 2011; 36(22):14923-14929.
[5] Shi Q, Diao G, Mu S. The electrocatalytic oxidation of glucose on the bimetallic Au-Ag particlesmodified reduced graphene oxide electrodes in alkaline solutions. Electrochimica Acta. 2014; 133:335-346.

[6] Brouzgou A, Song S, Tsiakaras P. Carbonsupported PdSn and Pd3Sn2 anodes for glucose electrooxidation in alkaline media. Applied Catalysis B: Environmetal 2014; 158-159:209216.

[7] Xin Y, Liu J-g, Zhou Y, Liu W, Gao J, Xie Y, Yin Y, Zou Z. Preparation and characterization of $\mathrm{Pt}$ supported on graphene with enhanced electrocatalytic activity in fuel cell. Journal Power Sources 2011; 196(3):1012-1018.

[8] Chakrabarti $\mathrm{MH}$ et al. Progress in the electrochemical modification of graphene-based materials and their applications. Electrochimica Acta 2013; 107:425- 440.

[9] Huang H, Chen H, Sun D, Wang X. Graphene nanoplate-Pt composite as a high performance electrocatalyst for direct methanol fuel cells. Journal of Power Sources 2012; 204:46-52.

[10] Dhara K et al. Pt-CuO nanoparticles decorated reduced graphene oxide for the fabrication of highly sensitive non-enzymatic disposable glucose sensor. Sensors and Actuators B: Chemical 2014; 195:197-205.

[11] Hummers Jr WS, Offeman RE. Preparation of Graphitic Oxide. Journal of the American Chemical Society. 1958; 80(6):1339.

[12] Kim JH et al. Influence of Sn content on PtSn/C catalysts for electrooxidation of $\mathrm{C} 1-\mathrm{C} 3$ alcohols: Synthesis, characterization, and electrocatalytic activity. Applied Catalysis B: Environmental 2008; 82(1-2):89-102.

[13] Marcano DC, Kosynkin DV, Berlin JM, Sinitskii A, Sun Z, Slesarev A, Alemany LB, Lu W, Tour JM. Improved Synthesis of Graphene Oxide. ACS Nano 2010; 4(8):4806-4814.

[14] Li Y, Tang L, Li J. Preparation and electrochemical performance for methanol oxidation of pt/ graphene nanocomposites. Electrochemistry Communications 2009; 11(4):846-849. 